\title{
Newborn screening for congenital hypothyroidism: Impact of parents counseling on the uptake of program.
}

1. M.Phil (Biochemistry)

Research Officer/Centre In-charge

PHRC Research Centre NHRC,

Shaikh Zayed Medical Complex

Lahore.

2. FCPS

Associate Professor Obstetrics and

Gynecology

Federal Postgraduate Medical Institute,

Shaikh Zayed Medical Complex Lahore.

3. M.Phil (Biotechnology)

Research Officer

PHRC Research Centre NHRC,

Shaikh Zayed Medical Complex Lahore.

4. M.Phil (Statistics), Ph.D Assistant Professor Lahore Business School,

The University of Lahore, Lahore.

Correspondence Address:

Saima Naz Mohsin

Research Officer/Centre In-charge

National Health Research Complex,

Shaikh Zayed Hospital Lahore.

shafqatzulfiqar@ymail.com

Article received on:

28/01/2020

Accepted for publication:

29/04/2020

\section{Saima Naz Mohsin ${ }^{1}$, Shafqat Zulfiqar ${ }^{2}$, Rabail Javed ${ }^{3}$, Amir Razi $^{4}$}

ABSTRACT... Objectives: The objectives of the study were to screen newborns for congenital hypothyroidism and to compare effects of two different modes of awareness on parent's consent directed to newborn screening for congenital hypothyroidism. Study Design: Cross-sectional study. Setting: Shaikh Zayed Hospital Lahore Pakistan. Period: 2018 to 2019. Material \& Methods: Both parents were counseled regarding the importance of newborn screening for $\mathrm{CHT}$. The statistics of program during current study (after parents counseling) were compared with study conducted during 2010 to 2011 (when parents were provided with printed awareness brochures). Results: Frequency of confirmed cases of CHT was 2.4 in 1000 babies screened for $\mathrm{CHT}$ during current study period. Proportion of parents consented for screening was $57.3 \%$ \& $41.1 \%$ respectively during two study periods. The proportion of parents consented for screening was increased by $16.2 \%$ subsequent to proper counseling, which is highly significant difference at $p<0.0001$. Conclusion: Proper counseling of both parents has improved the coverage of program. Therefore proper counseling session of parents regarding their baby's screening for congenital hypothyroidism should be conducted in order to improve their understanding and to emphasize the importance and benefits of screening.

Key words: $\quad$ Blood Spot TSH, Congenital Hypothyroidism, Newborn Screening, Parents Counseling.

Article Citation: Mohsin SN, Mukhtar S, Javed R, Razi A. Newborn screening for congenital hypothyroidism: impact of parents counseling on the uptake of program. Professional Med J 2020; 27(11):2350-2356. https://doi.org/10.29309/TPMJ/2020.27.11.4528

\section{INTRODUCTION}

Emphasis on the importance of newbornscreening (NBS) for congenital hypothyroidism $(\mathrm{CHT})$ is based on the fact that $\mathrm{CHT}$ during newborn period is almost overlooked due to absence of detectable physical signs, hence delayed diagnosis leads to the most severe outcomes. $\mathrm{CHT}$ is a preventable mental retardation which is caused by high TSH levels in newborn. ${ }^{1}$ Within the initial years of life (13 ) the brain development is greatly dependent on thyroid hormones. Any disturbances in this can lead into crucial life time abnormalities. Congenital hypothyroidism is mainly classified as transient or permanent type $\mathrm{CHT}$. The later type needs life time thyroidal treatment and is essentially due to primary hypothyroidism which is characterized with high TSH levels. ${ }^{2}$ Majority of cases are reported to have thyroidal dysgenesis while $20 \%$ cases are due to thyroid dyshormonogenesis.
Thyroid dyshormogenesis is associated with gene mutation in protein synthesizing thyroid hormone. ${ }^{3}$ New born does not shows evident clinical symptoms of hypothyroidism until they reach the age of 3 months with untreated/ undetected condition. ${ }^{4}$

In recent decade new born screening for congenital hypothyroidism is a program used in many countries all over the globe. ${ }^{1}$ Before NBS was introduced the incidence of $\mathrm{CHT}$ was 1:7K-10Kof live births. ${ }^{5}$ This incidence increased to 1: 3-4K live births after NBS started and are still increasing rapidly. ${ }^{6}$ In countries from America, New Zealand and Asia; the incidence has doubled during past years upto1:3985 (1987) to 1:2273 (2002) and 1:1415 (2005). ${ }^{7}$ This increase in incidence is due to early detection and better parents counseling of $\mathrm{CHT}^{8}$ 
There is a large variation between cut off assigned for TSH levels. Generally it is adapted that if new born sample is taken within 24 hours than cutoff is greater than $60 \mathrm{mIU} / \mathrm{L}$. However if the sample is withdrawn after 72 hours than the cutoff range is $>15 \mathrm{mIU} / \mathrm{L}$. Most of the established NBS program uses $20 \mathrm{mIU} / \mathrm{L}$ as their cutoff range..$^{3,9}$

NBS if properly established can prevent the chances of mental retardation in children. However unfortunately still $71 \%$ of babies born belong to those countries where a proper established NBS program for $\mathrm{CHT}$ is missing. ${ }^{10}$ Pakistan is in its initial stages for developing NBS for CHT. In a previous Pakistani study conducted in Sheikh Zayed hospital Lahore the incidence of NBS for CHT has been reported as 2:1357 cases ${ }^{5}$ which is alarmingly high and implicates the urgency of verification by a well refined new born screening program for $\mathrm{CHT}$ in this country.

Pakistan being a developing country has a low educational level within general population. Most of the people are unaware of health related preventives due to lack of awareness programs. Already held newborn screening programs for CHT in Pakistan faced many hurdles due to improper patients counseling, cost of test and lack of awareness about CHT and its consequences. The current study aimed to screen newborns for congenital hypothyroidism at Department of Gyn/Obs Shaikh Zayed Hospital Lahore and to demonstrate the effect of proper counseling of both parents on their consent directed to newborn screening for congenital hypothyroidism and to describe the statistics of coverage during current study period and compare it with that reported previously when mode of awareness was printed brochures.

\section{MATERIAL \& METHODS}

Cross-sectional study was carried out from March 2018 to April 2019 in Obstetrics/Gynecology department of Shaikh Zayed Hospital Lahore Pakistan. All newborns delivered (vaginally or by $\mathrm{C}$-section) during this period were part of the screening program, whereas those who were premature $(\mathrm{GA}<34$ weeks) were excluded. After delivery both parents were counseled by one of the authors, who thoroughly explained the importance as well as benefits of newborn screening program for congenital hypothyroidism $(\mathrm{CHT})$. Parents were provided with the following information in their local language:

- $\mathrm{CHT}$ is inborn metabolic disease in which a baby is unable to produce sufficient thyroxin; a hormone required for the normal growth and brain development

- If the condition is left undiagnosed and untreated, the baby can suffer with growth failure and irreversible mental retardation.

- The condition is absolutely preventable if it is diagnosed at birth and if the treatment is started in the first month of life

- The problem with its early diagnosis is that, the condition is asymptomatic at birth and the time when baby starts showing symptoms is too late to prevent or treat these lifelong sequelae

- The only option to deal with the problem is to screen every newborn for $\mathrm{CHT}$ at birth and start immediate treatment within first month of life.

Parents were also explained about the cost, method of taking blood spot samples of their babies, approximate time of getting results, and follow up plan for those babies showing positive screening results. A written informed consent form was signed by parents who agreed to get their baby screened.

A brief demographic history of mother and newborn was recorded on a pre-designed proforma. Information regarding mother's age, consanguineous marriage and history of taking thyroid medication were recorded. Information regarding baby's gestational age, gender, birth weight and any congenital anomalies in siblings was recorded on a separate proforma.

After 24 hours of baby's birth, blood spot samples were taken on filter paper through heel prick using sterile technique. Blood spot TSH was estimated using immuno-radiometric assay. All those newborns who had blood spot TSH value (>20 mlu/ml) were called back on day 7 of life 
for a full thyroid profile including serum TSH, FT3 and FT4. Results of follow up thyroid profile of newborns suspected positive for congenital hypothyroidism were provided to parents within 3 days. Confirmed cases of congenital hypothyroid were referred to pediatrician for appropriate treatment.

The coverage of newborns screened during current study following proper counseling of both parents at the time of screening, was compared with that reported previously from same hospital collected data from two hospital i.e. Shaikh Zayed Hospital \& Jinnah Hospital whereas data from Shaikh Zayed hospital was taken into comparison. ${ }^{9}$

For comparison information regarding total number of live births and hospital stay of these newborns during February 2010 to November $2011^{9}$ and March 2018 to April 2019 (current study) was obtained from records of Department of Obstetrics \& Gynecology Shaikh Zayed hospital Lahore. In both study, mothers of only those newborns were approached for their consent for newborn screening; whose stay in the hospital was more than 24 hours after birth.

For both studies under comparison, data on mode of awareness provided to mother/both parents, number of mothers consented or refused for newborn screening and total number of newborns got screened for CHT in Shaikh Zayed Hospital during the periods of both studies was obtained from records at National Health Research Complex.

Data was entered and analyzed using SPSS version 22.0. The association of between improved coverage of newborn screening for $\mathrm{CHT}$ and mode of awareness provided to the mothers was determined using Chi-square test. $\mathrm{P}<0.05$ was considered as significant.

\section{RESULTS}

\section{Statistics during current study period}

A total of 819 newborns were screened for congenital hypothyroidism (CHT) during March 2018 to April 2019. Out of 819 newborns 441 were males and 378 were females. Table-I shows number of newborns falling in different cutoff of blood spot TSH levels. Only 6 newborns showed blood spot TSH levels more than $20 \mathrm{mIU} / \mathrm{L}$ ranges from 27 to $96 \mathrm{mIU} / \mathrm{L}$ (Table-I).

\begin{tabular}{|c|c|c|c|}
\hline \multirow{2}{*}{$\begin{array}{c}\text { TSH cut off } \\
\text { mIU/L }\end{array}$} & \multicolumn{2}{|c|}{ Gender } & \multirow{2}{*}{ Total } \\
\hline$<5$ & 274 & 230 & 504 \\
\hline $5-10$ & 110 & 98 & 208 \\
\hline $11-20$ & 53 & 48 & 101 \\
\hline$>20$ & 4 & 2 & 6 \\
\hline Total & 441 & 378 & 819 \\
\hline Table-l. Distribution of newborns according to their \\
gender and blood spot TSH levels.
\end{tabular}

Six newborns suspected for CHT with blood spot TSH level more than $20 \mathrm{mIU} / \mathrm{L}$ were recalled for the confirmation of their thyroid status by evaluating complete thyroid profile using serum obtained through venipuncture. It was observed that 4 newborns showed normal thyroid profile and were confirmed as euthyroid, whereas 2 newborns initially suspected CHT on blood spot TSH screening were confirmed cases of $\mathrm{CHT}$ on serum thyroid profile at day 7 (Table-II). TableII shows complete picture of thyroid profile of newborns suspected positive on blood spot TSH screening. The frequency of confirmed cases of CHT was $0.24 \%$ (2 out of 819 ) newborns screened during this period, which is 2.4 in 1000 babies screened for $\mathrm{CHT}$.

\begin{tabular}{|l|c|c|c|c|c|}
\hline \multicolumn{1}{|c|}{ Diagnosis } & N & $\begin{array}{c}\text { Mean Blood Spot } \\
\text { TSH (m/U/L) }\end{array}$ & $\begin{array}{c}\text { Mean serum TSH } \\
\text { (m/U/L) }\end{array}$ & $\begin{array}{c}\text { Mean serum FT3 } \\
\text { (nmol/L) }\end{array}$ & $\begin{array}{c}\text { Mean serum FT4 } \\
\text { (nmol/L) }\end{array}$ \\
\hline Confirmed CHT & 2 & 88.5 & 99.5 & 0.15 & 0.98 \\
\hline Euthyroid & 4 & 30.6 & 2.8 & 2.9 & 14.5 \\
\hline \multicolumn{2}{r}{ Table-II. Serum thyroid profile of newborns suspected positive for CHT at initial blood spot TSH screening. } \\
\hline
\end{tabular}


Demographic as well as clinical features of confirmed cases of $\mathrm{CHT}$ are shown in table 3 . There were no remarkable findings that were observed as common in both cases and may be related to the status of hypothyroidism among these two cases. One confirmed female case of CHT was referred to pediatrician for treatment. One male baby who has been sick since his birth due to other anomalies was also confirmed as positive for $\mathrm{CHT}$. The baby expired at the age of 22 days (Table-III).

\section{Comparison of coverage of newborn screening program during two study periods.}

The coverage of newborns screened during current study following proper counseling of both parent's regarding importance, benefits and procedure of screening done at the time of screening was compared with that reported previously by Ghafoor et $\mathrm{al}^{9}$, who attached information brochure (translated in local language)to the antenatal cards of mother during their visit to antenatal clinic of same hospital.

\begin{tabular}{|l|c|c|}
\hline \multicolumn{1}{|c}{ Variables } & \multicolumn{2}{c|}{ Characteristics of Confirmed Cases of CHT } \\
Baby 2 \\
Baby 1
\end{tabular}

Statistics during both study periods were documented from records of Obs/Gynea department of Shaikh Zayed hospital Lahore and National health research complex (where studies were conducted) and described in Table-IV.

Table-IV shows that average number of deliveries per month (165 vs 171) and proportion of newborns dropped out (45.9\% vs $40.1 \%)$ due to early discharge before 24 hours after delivery was almost same during both study periods. Further it was observed that although screening was offered free of cost previously ${ }^{9}$ as compare to current study but the number of newborns (59.5\% increase).

Furthermore the awareness methods used for obtaining consent of mothers for newborn screening were compared and their effect on the uptake of program was assessed (Table-V). screened per year was increased from 440 to 702

Table-III. Demographical and clinical characteristics of confirmed cases of congenital hypothyroidism (CHT).

\section{Parameters for comparison}

Mode of Awareness provided to mothers regarding program
Ghafoor et al $^{9}$ Information brochure attached to antenatal cards of pregnant women attending antenatal OPD

Screening cost paid by parents

Study duration (months)

Total number of deliveries during study period

Average deliveries per month

No. of newborns discharged within 24hours after delivery (Could not accessed)

No. of newborns stayed in hospital for more than 24hours after delivery (Accessed for screening)

No. of newborns screened during study period

No. of babies Screened for CHT per year at Shaikh Zayed Hospital

Frequency of positive CHT /1000 newborns screened of hospital

\section{Current study}

Counseling of both parents was done by one of the authors and importance and benefits of screening was emphasized

\begin{tabular}{|c|c|}
\hline No & Yes \\
\hline 22 & 14 \\
\hline 3630 & 2388 \\
\hline 165 & 171 \\
\hline
\end{tabular}

1665 (45.9\%)

$958(40.1 \%)$

$1965(54.1 \%)$

$1430(59.9 \%)$

\begin{tabular}{|r|r}
808 & 819
\end{tabular}

440

1.47

702

2.44

Table-IV. Parameters under comparison during two study periods. 


\begin{tabular}{|l|c|c|}
\hline \multirow{2}{*}{$\begin{array}{c}\text { Parents consented for } \\
\text { Newborn Screening }\end{array}$} & $\begin{array}{c}\text { Mode of Awareness } \\
\text { Brochures attached to antenatal } \\
\text { cards of mothers for their } \\
\text { information (Ghafoor et.al. 2013) }\end{array}$ & $\begin{array}{c}\text { Counseling of both parents } \\
\text { (Current Study) }\end{array}$ \\
\hline Yes & $808(41.1 \%)$ & $819(57.3 \%)$ \\
\hline No & $1157(58.9 \%)$ & $611(42.7 \%)$ \\
\hline Total & 1965 & 1430 \\
\hline \multicolumn{2}{|c|}{ Table-V. Effect of mode of awareness on parent's consent for newborn screening. } \\
\end{tabular}

Proportion of parents consented for their newborns to be screened for congenital hypothyroidism was $57.3 \%$ when mode of awareness was a proper counseling session of both parents at the time of screening, which is different from proportion of parents (41.1\%) consented for newborn screening, when the mode of awareness was an informational brochure that was provided to the mother at the time of her antenatal visit at outpatient department of hospital. The proportion of parents consented for newborn screening subsequent to proper counseling was improved by $16.2 \%$, which is highly significant difference at $\mathrm{p}<0.0001$ (Table-V). This showed that counseling of both parents has improved the uptake of screening program.

\section{DISCUSSION}

Pakistan like other developing countries suffers from iodine deficiency causing life lasting anomalies in children. The incidence of congenital hypothyroidism has rapidly increased in Pakistan within the last decades. For this purpose early detection is most efficient management strategy through proper screening method. In the present study, a rise in incidence rate in $\mathrm{CHT}$ cases was estimated by comparing it with past screening results in a similar setting. The results showed frequency of $\mathrm{CHT}$ as $0.24 \%$ than previously reported $0.14 \% .{ }^{9}$ This difference was caused by enrolling larger number of participants than before, consequently providing a better incidence status of $\mathrm{CHT}$ within Pakistan. Literature supports that number of deliveries has also increased in Asian Populations in last decade therefore ascending the number of newborns born with congenital hypothyroidism. ${ }^{10}$

In this study there were two newborn children confirmed for $\mathrm{CHT}$ later by TSH follow up. Out of these one was a male child and the other was a female. Studies report that CHT caused by dysgenesis is more common in female children while dyshormonogenesis is presented equally in both genders. ${ }^{11}$ Researches elsewhere reported that the chances of $\mathrm{CHT}$ were more common in post term deliveries ${ }^{12}$, however in this study both newborns had a normal gestation age with none other siblings involved. It is not necessary that siblings of CHT newborn should also suffer from this condition. ${ }^{13}$ The male baby was diagnosed with congenital cardiac dysfunctioning, low birth weight and could unfortunately not survive after 22 days of hospitalization. Likewise further studies reports low birth weight and associated anomalies to be presented in CHT cases..$^{10,11,14}$ The family history of $\mathrm{CHT}$ diagnosed male baby, confirmed consanguineous marriage. Consanguinity is predominant in Middle East and Asian Countries. ${ }^{15}$ Researchers reported a highly significant association $(>31 \%)$ between consanguineous marriages and congenital disorders. ${ }^{16,17}$

Awareness Campaigns are the best solution for increasing participant enrollment in any disease outbreak and screening program. In the past $\mathrm{CHT}$ screening was done by taking consent of mothers only with brochures displayed in gynae ward. ${ }^{9}$ This did not aware the parents properly of the disease and its consequences. In the current study counseling of both parents (instead of only mothers) for enhanced awareness of $\mathrm{CHT}$ was conducted resulting in devastating acceleration in CHT screening enrollment. Studies report that in children, any disease eradication is significantly dependent on parental awareness and counseling. A proper communication helps 
parents better understand the reasoning behind implication of health program. ${ }^{18,19}$ In current study counseling of both parents increased the consent rate of $\mathrm{CHT}$ screening from $41.1 \%{ }^{9}$ to $57.3 \%$. The rise in enrollment rate is also dependent on screening all newborn delivered. Unfortunately the present study could not enroll newborns discharged within 24 hour of their delivery. However new strategies are being developed to overcome this problem by taking cord blood instead of heel prick. This cord blood can be achieved just after birth, therefore this method would be able to target those discharged within 24 hours after delivery. The cord blood TSH is as reliable as heel prick TSH results of newborns. ${ }^{20}$

\section{CONCLUSION}

Mode of awareness provided to parents regarding importance of newborn screening program affects their consent for their baby's screening. Proper counseling of both parents has improved the coverage of program.

\section{Practice Implication}

Proper counseling session of parents concerning their baby's screening for congenital hypothyroidism should be conducted in order to improve their understanding and to emphasize the importance of screening. This practice can increase the coverage of newborn screening program.

\section{Copyright(C) 29 Apr, 2020.}

\section{REFERENCES}

1. NoumanA, Asral, Saad SA. Congenital hypothyroidism: Screening, diagnosis, management, and outcome. J Clin Neonatol 2017; 6:64-70.

2. RastogiRM, LaFranchi SH. Congenital hypothyroidism. Orphanet. J Rare Dis 2010; 5: 17.

3. Léger J, Olivieri A, Donaldson M, Torresani T, Krude $H$, van Vliet $G$, et al. European Society for Paediatric Endocrinology consensus guidelines on screening, diagnosis, and management of congenital hypothyroidism. J Clin Endocrinol Metab 2014; 99: 363-84.

4. Vulsma T, Gons MH, de Vijlder JJ. Maternal-fetal transfer of thyroxine in congenital hypothyroidism due to a total organification defect or thyroid agenesis. N Engl J Med 1989; 321:13-6.
5. Alm J, Larsson A, Zetterström R. Congenital hypothyroidism in Sweden. Incidence and age at diagnosis. Acta Paediatr Scand 1978; 67: 1-3.

6. Delange F. Neonatal screening for congenital hypothyroidism: Results and perspectives. Horm Res 1997; 48: 51-61.

7. Harris KB, Pass KA. Increase in congenital hypothyroidism in New York State and in the United States. Mol Genet Metab 2007; 91: 268-77.

8. LaFranchi $\mathrm{SH}$. Newborn screening strategies for congenital hypothyroidism: An update. J Inherit Metab Dis 2010; 33: S225-33.

9. Ghafoor F, Mohsin SN, Mukhtar S, Younas S, Hussai W. Newborn screening for congenital hypothyroidism in a public sector hospital. Pak J Med Res 2013; 52: 3942.

10. Harris $\mathrm{KB}$, Pass $\mathrm{KA}$. Increase in congenital hypothyroidism in New York State and in the United States. Mol Genet Metab 2007; 91: 268-77.

11. http://www.btf-thyroid.org/information/leaflets/42congenital-hypothyroidism-guide.

12. Mao $H Q$, Yang RL, Liu ZH. Correlation of congenital hypothyroidism with birth weight and gestational age in new born infants. Zhejiang Da Xue Xue Bao Yi Xue Ban 2007; 36: 378-81.

13. https://www.newbornscreening.info/Parents/ otherdisorders/CH.htmI\#11

14. Ng SM, Wong SC, Paize F, Chakkarapani E, Newland P, Isherwood D, Didi M. Multivariate analyses of factors that affect neonatal screening thyroid stimulating hormone. J Pediatr Endocrinol Metab 2011; 24: .727-32

15. Bener A, Hussain R. Consanguineous unions and child health in the State of Qatar. Paediatr Perinat Epidemiol 2006; 20: 372-8.

16. Shawky RM, Elsayed SM, Zakie ME, Nour el-Din SM, Kamal FM. Consanguinity and its relevance to clinical genetics. Egypt J Med Hum Genet 2013; 14: 157-64.

17. Kanaan ZM, Mahfouz R, Tamim H. The prevalence of consanguineous marriages in an underserved area in Lebanon and its association with congenital anomalies. Genet Test 2008; 12: 367-72.

18. Al-lela OQ, Bahari MB, Al-Qazaz HK, Salih MRM, Jamshed SQ, Elkalmi RM. Are parents' knowledge and practice regarding immunization related to pediatrics' immunization compliance? A mixed method study. BMC Pediatr 2014; 14:20. 
19. Gellin B, Maibach E, Marcuse E. Do parents understand immunizations? A national telephone survey. Pediatrics 2000; 106: 1097-1102.
20. Anju S, Rashmi M, Bhakhri BK, Sekri T. Neonatal thyroid screening: relationship between cord blood thyroid stimulating hormone levels and thyroid stimulating hormone in heel prick sample on 4th to 7th day-oflife. Indian J Endocrinol Metab 2014; 18: 125-6.

\begin{tabular}{|c|c|c|c|}
\hline \multicolumn{3}{|c}{ AUTHORSHIP AND CONTRIBUTION DECLARATION } \\
\hline Sr. \# & Author(s) Full Name & \multicolumn{1}{|c|}{ Contribution to the paper } \\
\hline 1 & Saima Naz Mohsin & \begin{tabular}{l} 
Conception and design of the study, The \\
acquisition of data, Final interpretation of \\
newborn screening test resutls and made \\
laboratyory diagnosis, Drafted manuscript \\
and interpretation of data. \\
Counseling of mother and taking consents \\
for newborn screening, Provided revision \\
and final approval of the version to \\
published. \\
Drafted manuscript, Provided critical \\
revision of the article. \\
\hline 3
\end{tabular} & $\begin{array}{l}\text { Shafqat Zulfiqar } \\
\text { Data entry and cleaning, Final statistical } \\
\text { analysis and interpretation of data. }\end{array}$ \\
\hline 4 & Amir Razi & Rabail Javed
\end{tabular}

